\title{
Canadian Association of Gastroenterology Practice Guidelines: Evaluation of abnormal liver enzyme tests
}

Gerald Y Minuk MD FRCPC

\begin{abstract}
A pproximately one in 10 Canadians has at least one abnormal liver biochemical test on routine screening. Although in the majority of these individuals the abnormality reflects a benign liver condition such as Gilbert's syndrome or enzyme induction from recent alcohol consumption, a subgroup will have progressive and potentially life-threatening liver disease for which therapeutic interventions are often available (1). The objectives of these guidelines are to, first, outline when screening for liver disease would be appropriate; second, determine which biochemical tests should be used in screening for liver disease; third, review second-line tests for when screening tests are positive; fourth, outline when referrals to specialists would be appropriate; and, fifth, review additional testing that specialists might undertake in their evaluation of the patient.

Because sanctioned guidelines for investigating asymptomatic patients with liver biochemical abnormalities have not been developed, the options, outcomes and evidence considered in formulating the present guidelines largely reflect the experience of individuals with expertise in the area and a limited number of clinical studies in which liver biochemical abnormalities were described in the healthy blood donor population as well as in patients with various forms of acute and chronic liver disease.
\end{abstract}

SPONSORS AND VALIDATION
This practice guideline was developed by
Dr Gerald Y Minuk MD FRCPC and was reviewed by
- Practice Affairs Committee (Chair -
Dr A Cockeram): Dr T Devlin, Dr J McHattie,
Dr D Petrunia, Dr E Semlacher and Dr V Sharma
- Canadian Association of Gastroenterology (CAG)
- The Canadian Association for the Study of the
Liver (CASL) - Dr Gary Levy and Dr Eve Roberts
- CAG Governing Board

\section{BENEFITS, HARMS AND COSTS}

The benefits, harms and costs of screening for liver disease can only be estimated on the basis of efficiently arriving at a correct diagnosis (or at least appreciation) of the general form of liver disease (hepatocellular versus cholestatic), avoidance of unnecessary, interventional procedures and efficient use of laboratory testing.

University of Manitoba, Liver Diseases Unit, Winnipeg, Manitoba

Correspondence and reprints: Dr GY Minuk, Professor of Medicine 8 Pharmacology, University of Manitoba, Director, Liver Diseases Unit, GF 407-Health Science Centre, 820 Sherbrook Street, Winnipeg, Manitoba R3A 1R9. Telephone 204-787-4662, fax 204-775-4255, e-mail gminuk@cc.umanitoba.ca 


\section{RECOMMENDATIONS}

When to screen for liver disease: Screening for evidence of liver disease should be performed in the following instances:

- during routine annual evaluations of patients in all age groups but particularly those with risk factors for liver disease (see below) including obesity, diabetes and hyperlipidemia (conditions associated with fatty liver);

- in patients with a history of high risk activities, such as intravenous drug use, sexual promiscuity, homosexual contacts, tattoos, nonsterile ear or body piercing, extensive travel or residence in developing nations, previous blood or blood product transfusions, ongoing use of prescribed or excess amounts of over-the-counter medications, alcohol abuse, employment or hobby exposure to chemicals and/or toxins in nonventilated rooms and past history of autoimmune disorders or family history of liver disease;

- in patients with a history of unexplained right upper quadrant discomfort, dark urine, pale stool or jaundice;

- in patients with the following physical findings: unexplained weight loss, scleral icterus or jaundice, more than four spider nevi in females and two in males, hepatomegaly, bruits or rubs in the right upper quadrant, unexplained splenomegaly, flank distention and/or shifting dullness, extensive superficial abdominal veins, peripheral edema, palmar erythema, Terry's nails (proximal $80 \%$ of the nail bed is white rather than pink, associated with cirrhosis), Dupuytren's contractures and impaired cognitive function.

Screening for liver disease: In addition to a complete history and physical examination, the following tests are recommended for excluding the presence of liver disease: serum alanine aminotransferase (ALT), aspartate aminotransferase (AST) and alkaline phosphatase (ALP). The rationale for each of these tests is as follows.

ALT: The ALT is both sensitive and specific for liver disease of a hepatocellular injury type. Elevations in ALT levels should be interpreted as indicative of liver disease with only rare exceptions: severe rhabdomyolysis or systemic myopathies. While ALT levels can be normal in the presence of liver disease (most often seen in patients with alcoholinduced liver disease or inactive cirrhosis), on those occasions additional screening tests usually identify the presence of liver disease (AST abnormalities in the setting of alcoholinduced liver disease) or reveal that therapeutic interventions are not warranted (in the setting of inactive cirrhosis). One common exception is patients with liver disease associated with methotrexate use; another less common exception comprises a subpopulation of chronic hepatitis $\mathrm{C}$ virus (HCV) carriers. In both these conditions biochemical screening tests can be negative yet active liver disease is present histologically $(2,3)$. In those clinical scenarios, additional investigations are warranted (liver biopsy and viral serology, respectively).
AST: The AST enzyme is less sensitive and specific for liver disease but still should be employed as a screening test because the ALT to AST ratio can often be used to suggest the cause and/or extent of liver disease. For example, ALT to AST ratios greater than 1 are typically found in patients with viral hepatitis, drug-induced liver disease, autoimmune disorders, etc, whereas ratios less than 1 are more often associated with alcohol-induced liver disease, ischemic forms of liver disease (passive congestion or under perfusion), biliary tract obstruction and certain disorders that tend to result in a predominantly mitochondrial form of cell injury such as fatty liver of pregnancy, tetracycline toxicity, Reye's syndrome, etc. The use of this ratio can also be helpful when assessing the severity of liver disease because once liver disease has progressed to cirrhosis (regardless of the underlying etiology) a previously elevated ALT to AST ratio often falls to values of 1 or less (4). It should be noted that in patients with acute, severe forms of hepatocellular injury (aminotransferase values in excess of $1000 \mathrm{IU} / \mathrm{L}$ ) the ALT to AST ratio is not of diagnostic value.

ALP: By definition, serum ALP levels are elevated in all forms of cholestatic liver disease. However, levels can also be elevated in pregnancy, in patients with bone disease and, on occasion, inflammatory bowel disorders in the absence of hepatobiliary disease (5). Low or undetectable levels of ALP have only rarely been described in patients with an acute onset of Wilson's disease (6).

Second-line biochemical testing: All patients with at least one abnormal liver screening test (abnormal ALT, AST or ALP) should have the following liver biochemical tests performed: gamma-glutamyl transferase (GGT), albumin, bilirubin (including direct if the total bilirubin is elevated) and either prothrombin time (PT) or international normalized ratio (INR). These tests can also be performed as initial screening tests if it is inconvenient for the patient to return to the physician's office within a reasonable period of time (weeks or months depending on the severity of the enzyme abnormalities).

The GGT test is useful in confirming that elevated ALP levels reflect hepatobiliary disease. Only in some pregnant women with liver disease will GGT values be normal despite the presence of underlying cholestasis (5). In these rare instances, fractionation of the ALP is warranted. Due to the ease and frequency with which GGT values can become elevated (recent ingestion of alcoholic beverages, concurrent use of antiseizure medication, etc) and that it can be elevated in nonhepatobiliary disorders (renal disease, pancreatitis, diabetes, coronary artery disease and carcinoma of the prostate), this test should not be used as a screening test for liver disease. However, if for some reason it has been performed as part of the initial screening for liver disease and is found to be the only abnormality present, in the absence of the conditions listed above it is reasonable to reassure the patient that as many as $10 \%$ of the general population have elevated GGT levels yet have no underlying hepatobiliary disease or cause for concern.

Serum albumin, bilirubin and PT/INR document whether 
the liver disease is of sufficient severity that hepatic function is impaired (7). In the setting of abnormal screening tests, these tests usually reflect hepatic dysfunction. Because each test can be abnormal in the absence of liver disease (eg, hypoalbuminemia with malnutrition, hyperbilirubinemia with hemolysis and prolonged PT with vitamin K deficiency states), they should not be used as screening tests.

Interpretation of liver biochemical abnormalities: Once the presence of liver disease is established, the next step is to determine whether the disease is of a hepatocellular injury type (hepatitis) or is a cholestatic liver disorder. In the former, aminotransferase abnormalities predominate whereas in the latter, the ALP abnormality is more apparent. Similar elevations (eg, aminotransferase values four times normal and ALP levels four times normal) tend to suggest cholestatic rather than hepatocellular forms of liver disease. Thus, only when aminotransferase elevations are twice those of ALP enzyme elevations or greater (eg, AST or ALT eight times normal versus ALP four times normal), would the problem be considered predominantly hepatocellular.

As indicated above, liver enzyme abnormalities associated with biochemical evidence of hepatic dysfunction (albumin, bilirubin or PT/INR abnormalities) suggest that the disease is advanced and hepatic function is compromised.

When to refer to specialists: Consultation with physicians with expertise in hepatobiliary disorders is suggested for patients with unexplained liver enzyme abnormalities greater than 1.5 times normal on two occasions, a minimum of six months apart; patients with explained liver disease associated with evidence of hepatic dysfunction (hypoalbuminemia, hyperbilirubinemia, prolonged PT or INR); and in patients with explained liver disease where treatment beyond withdrawal of the implicated agent (alcohol or drugs) is warranted. The latter group of patients might include those who require antiviral therapy for chronic viral hepatitis, immunosuppressive agents for autoimmune liver disorders, ursodeoxycholic acid for nonalcoholic steatohepatitis (fatty liver), phlebotomy or chelation therapy for hemochromatosis, etc. The following tests should be considered before referral to a specialist.

In patients with biochemical evidence of a hepatocellular disorder, the following tests should be preformed: viral hepatitis screening (immunoglobulin [Ig] M anti-hepatitis A virus [HAV], hepatitis B surface antigen $[\mathrm{HBsAg}]$ and anti$\mathrm{HCV}$ ); antinuclear antibody levels; ceruloplasmin levels in patients younger than 40 years old; iron studies (iron, total iron binding capacity and ferritin levels) in individuals older than 40 years old, or in those with chronic hemolytic anemia or other blood disorders that require ongoing blood transfusions; and an ultrasound in individuals where fatty infiltration of the liver is suspected (obese, diabetic and/or hyperlipidemic patients). Ultrasound should also be performed when the patient has liver enzyme abnormalities, is symptomatic (right upper quadrant pain/tenderness, fever, chills, nausea, vomiting, etc) or has evidence of hepatic dysfunction (increased bilirubin or PT, or decreased albumin).
In patients with biochemical evidence of a cholestatic form of liver disease, an ultrasound of the abdomen should be performed.

\section{ADDITIONAL TESTING OFTEN PERFORMED BY A SPECIALIST}

The following are considered appropriate additional tests for patients with liver enzyme abnormalities in certain circumstances.

Positive for IgM anti-HAV: Because a positive test for IgM anti-HAV is sufficient to diagnosis acute HAV infection, no additional diagnostic testing is required.

Positive for HBsAg: To determine whether the positive HBsAg reflects an acute or chronic infection an IgM antibody to hepatitis B core antigen (IgM anti-HBc) test should be performed. If the result is positive (suggesting an acute HBV infection), the HBsAg and antibody to HBsAg (antiHBs) should be obtained at six months to document whether the acute infection has evolved into a chronic infection (HBsAg positive, anti-HBs negative) or has resolved (HBsAg negative, anti-HBs positive). On the other hand, if the IgM anti-HBc is negative (suggesting a chronic HBV infection), a hepatitis $\mathrm{B}$ e antigen ( $\mathrm{HBe} \mathrm{Ag}$ ) and antibody to $\mathrm{HBeAg}$ (anti-HBe) should be obtained to determine whether the carrier's infection is actively replicating or quiescent. If the former, consideration should be given to antiviral therapy. An HBsAg-positive individual's family should also be screened for evidence of HBV exposure (initial screening test, anti-HBc). Anti-HBc-negative family members and/or intimate contacts would be candidates for immunoprophylaxis if not previously vaccinated for HBV.

Positive for anti-HCV: If the initial screening test for HCV was performed by an enzyme immunoassay and not confirmed by additional testing (radioimmunoblot assay or HCV RNA testing), then confirmatory testing should be obtained. If the confirmatory test is negative then gammaglobulin levels and a rheumatoid factor should be obtained to help explain the apparent false-positive anti-HCV screening test. If the confirmatory tests are positive, repeat abnormal liver enzyme test results six months later will confirm the presence of chronic infection. If the results are elevated on both occasions, a liver biopsy will assist in determining whether antiviral therapy is required. A biopsy is also helpful in predicting the response therapy; advanced disease with extensive fibrosis is less likely to respond to presently available therapy than early disease without fibrosis. The presence of cirrhosis warrants treatment only in the context of clinical trials or in consultation with a specialist. In general, liver biopsies should be considered helpful but not essential for the treatment of chronic $\mathrm{HCV}$ infections. If the follow-up liver enzyme results are normal, an HCV-RNA test should exclude the unlikely possibility that the infection has spontaneously resolved. If the HCVRNA test is positive then a liver biopsy should be obtained. On the other hand, if the RNA test is negative, it should be repeated six months later and then one year thereafter; if negative and with consistently normal liver enzymes on all three occasions, then the infection can be considered resolved. 
Positive for antinuclear antibodies: To help support a diagnosis of autoimmune chronic hepatitis (particularly when the antinuclear antibody is only weakly positive), an antismooth muscle titre, $\mathrm{C} 3$ and $\mathrm{C} 4$ complement levels and protein electrophoresis or gammaglobulin levels should be obtained. When the problem appears to have been a longstanding one and the liver disease is advanced (autoantibodies tend to wane and often become negative in this setting), human leukocyte antigen typing (for haplotypes B8 Dr3 or 4) can be useful (8).

Low ceruloplasmin: A $24 \mathrm{~h}$ urine copper level (collected in a copper-free container) should be obtained to help support the diagnosis of Wilson's disease (9). If the urine copper level is significantly elevated (mild elevations of two to three times normal are common in many forms of liver disease), blood should be sent for genetic screening (if available) and a biopsy of the liver should be obtained for copper quantitation. Siblings should also be screened for evidence of Wilson's disease (serum ceruloplasmin or genetic screening if available). A low serum ceruloplasmin in the setting of normal or minimally elevated urinary copper excretions is more in keeping with decreased synthetic function of the liver.

Abnormal iron studies: If the iron saturation (ratio of iron to total iron binding capacity) is greater than $60 \%$ and ferritin levels exceed 10 times the upper limits of normal, a liver biopsy should be obtained for iron staining and quantitation (10). If the results are in keeping with idiopathic hemochromatosis, screening of siblings should be performed, with tests to include iron and ferritin levels, total iron-binding capacity and genetic screening if available. If genetic screening is not available, human leukocyte antigen typing can determine which siblings are at greatest risk of developing hemochromatosis. Genetic screening for the C282Y gene is positive in more than $90 \%$ of patients with biopsy-confirmed hemochromatosis. Preliminary results from population-based studies indicate a one in 250 gene frequency.

Initial diagnostic screening tests negative and patient known to be obese, diabetic and/or hyperlipidemic: An ultrasound of the abdomen should be obtained in obese, diabetic and/or hyperlipidemic patients to exclude nonalcoholic steatohepatitis. If fatty infiltration is documented then the underlying metabolic abnormality should be corrected. If the enzymes remain abnormal then a liver biopsy should be done. If the ultrasound fails to identify fatty infiltration and the aminotransferase values exceed 2.5 times normal, it is appropriate to correct the underlying metabolic abnormality (false negative ultrasounds for fatty liver occur in $10 \%$ to $30 \%$ ); if the enzymes remain elevated, proceed to a liver biopsy.

Cholestatic enzyme abnormalities with ductal dilation on ultrasound: Patients with cholestatic enzyme abnormalities with ductal dilation on ultrasound should proceed directly to endoscopic retrograde cholangiopancreatography or percutaneous transhepatic cholangiography to identify and relieve the cause of the obstruction. This approach also applies to patients with cholestatic liver enzyme abnormalities and a previous cholecystectomy (which can be associated with mild ductal dilation).
Cholestatic enzyme abnormalities and no ductal dilation but space occupying lesions present: Additional testing in patients with cholestatic enzyme abnormalities and no ductal dilation but space occupying lesions present, depends on the patient's clinical presentation. If an underlying malignancy is suspected and a hemangioma has been excluded (often by the initial ultrasound or with subsequent red blood cell scans and/or computerized tomography) a liver biopsy should be obtained, preferably under ultrasound guidance. If the lesion is cystic and there is evidence on history that echinococcal exposure has occurred, echinococcal serology should be obtained before the biopsy/aspiration. Simple cysts and polycystic disease of the liver do not require additional investigations. Cholestatic liver disease without ductal dilation or space occupying lesions: Mechanical obstruction of the biliary tract without ductal dilation can occur in the following cases: during the acute phase of the obstruction (it may take 6 to $12 \mathrm{~h}$ for the ducts to dilate following stone impaction); with intermittent obstructions (eg, passed stones); and in sclerosing cholangitis where the ducts are too fibrotic and rigid to dilate. Having considered these conditions, the remaining causes can be divided into intrinsic diseases, infiltrative disorders and systemic disease without hepatic involvement (11). In intrinsic diseases of the liver, atypical presentations of alcoholic liver disease, drugs, certain viral infections, primary biliary cirrhosis, primary sclerosing cholangitis and autoimmune cholangitis should be considered. A careful history is usually all that is required to exclude an atypical presentation of alcohol-induced liver disease because the majority of these patients are heavy alcohol users (12). All unnecessary drugs and particularly those started within the past three months should be held to determine whether the liver enzyme abnormalities resolve with follow-up. The two viral infections that most often present with a significant cholestatic component are HCV and, in the immunocompromised host, cytomegalovirus infections. These can be identified by anti-HCV, and by CMV DNA (if available), IgM anticytomegalovirus, urine cytomegalovirus or shell vile testing, respectively. A mitochondrial antibody is usually sufficient to diagnose primary biliary cirrhosis. Perinuclear antineutrophil cytoplasmic antibodies are often helpful in diagnosing primary sclerosing cholangitis when the patient's presentation is atypical (no history of inflammatory bowel disease). Antinuclear antibodies should be obtained to exclude autoimmune cholangitis.

Infiltrative disorders of the liver that should be considered include granulomatous infiltration and hematological malignancies. A chest $\mathrm{x}$-ray is usually sufficient to exclude the former (tuberculosis or sarcoidosis); the diagnosis of a hematological malignancy is typically made before patients present with asymptomatic liver enzyme abnormalities.

The systemic disorders most often associated with cholestatic enzyme abnormalities are often readily apparent (pregnancy, sepsis, etc) and do not require further investigations unless the enzyme abnormalities persist after the systemic disorder has resolved. Occult, pyogenic abscesses are an exception. Careful history and physical examination should be 
obtained, and body temperature throughout the day should be monitored (13).

Indications for liver biopsy: In general, liver biopsies should be obtained in the following settings (14-16):

- in patients with unexplained, persistent (beyond six months) hepatocellular enzyme abnormalities where the aminotransferase values exceed 2.5 times normal (or less when the patient is symptomatic);
- in patients with unexplained cholestatic liver disease of any magnitude or duration beyond three months;

- to confirm a diagnosis when blood work and/or radiologic imaging is not conclusive; or

- when the results will assist in decisions on initiation and/or maintenance of therapy.

\section{Canadian Association of Gastroenterology Practice Guideline Disclaimer}

This clinical practice guideline has been developed by the author on behalf of the Canadian Association of Gastroenterology (CAG) in order to outline the clinical approach to management problems or training issues. After preparation by the author, based on a review of the literature, each guideline is extensively reviewed by the CAG Practice Affairs Committee, composed of practitioners from across Canada. Changes are made, and once the guideline is felt to be appropriate, it is then circulated for further review by recognized Canadian experts and then amended further. Finally, the guideline is presented to the CAG Governing Board for further review and final approval.

Practice guidelines are intended to give an understanding of a clinical problem and outline one or more preferred approaches to investigation and management of the problem. While practice guidelines are intended to be useful to all physicians, it is recognized that specialists may rely less on practice guidelines than those in more general practice. These guidelines are intended to give a practical approach to a problem based on the current literature, but are not intended to be state-of-the-art reviews with extensive references.

Practice guidelines are developed to be of assistance to practising clinicians and are not intended to be the only approach to the management of clinical problems, nor are they intended to be considered as a 'standard of care'. The CAG Practice Affairs Committee recognizes that clinical circumstances may at times justify an approach different from that outlined in a practice guideline. It is also recognized that new developments in medical research and clinical practice may require subsequent changes to the practice guideline.

\section{REFERENCES}

1. Hav JE, Czaja AI, Rakela J, Ludwig J. The nature of unexplained chronic aminotransferase elevations of a mild to moderate degree in asymptomatic patients. Hepatology 1989;9:193-7.

2. Weinstein GD. Methotrexate. Ann Intern Med 1977;86:199-204.

3. Haber MM, West AB, Haber AD, Reuben A. Relationship of aminotransferases to liver histological status in chronic hepatitis $\mathrm{C}$. Am J Gastroenterol 1995;90:1250-7

4. Williams ALB, Hoofnagle JH. Ratio of serum aspartate to alanine aminotransferase in chronic hepatitis. Gastroenterology $1988: 95: 734.9$

5. Chopra S, Griffin PH. Laboratory tests and diagnostic procedures in evaluation of liver disease. Am J Med 1985;79:221-30.

6. Shaver WA, Bhatt H, Combes B. Low serum alkaline phosphatase activity in Wilson's disease. Hepatology 1986;6:859-63.

7. Minuk GY. Liver function tests. Med North Am 1985:21:2732.43.

8. Krawitt EL. Autoimmune hepatitis. N Engl J Med $1996: 334: 897.904$

9. Stremmel W, Meyerrose K.W, Niederau C, Hefter H, Kreuzpaintner G, Strohmever G. Wilson disease: clinical presentation, treatment, and survival. Ann Intern Med 1991:115:720-6.
10. Smith LH Jr. Overview of hemochromatosis. West J Med $1990: 153: 296-308$.

11. Frank BB, Members of the Patient Care Committee of the American Gastroenterological Association. Clinical evaluation of jaundice. A guideline of the patient care committee of the American Gastroenterological Association. JAMA 1989;262:3031-4.

12. Minuk GY, Thompson W. Biochemical features of alcohol-induced liver disease in native versus non-native Canadians. Can J Gastroenterol 1990:4:163-6.

13. Fang MH, Ginsberg AL, Dobbins WO III. Marked elevation in serum alkaline phosphatase activity as a manifestation of systemic infection. Gastroenterology 1980;78:592-7.

14. Czaja AJ, Carpenter HA. Sensitivity, specificity, and predictability of biopsy interpretations in chronic hepatitis. Gastroenterology $1993 ; 105: 1824.82$

15. Jacobs WH, Goldberg SB and the Patient Care Committee of the American Gastroenterological Association. Statement on outpatient percutaneous liver biopsy. Dig Dis Sci 1989;34:322-3.

16. Van Ness MM, Diehl AM. Is liver biopsy useful in the evaluation of patients with chronically elevated liver enzymes? Ann Intern Med $1989 ; 111: 473-8$. 


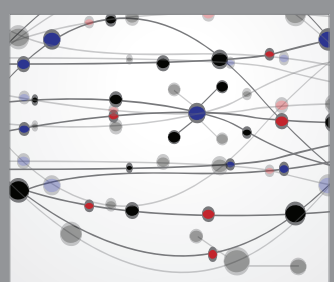

The Scientific World Journal
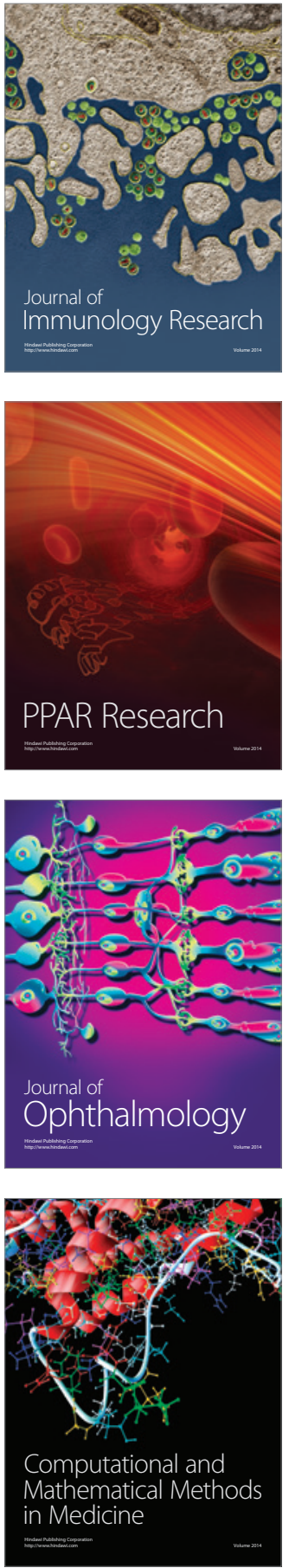

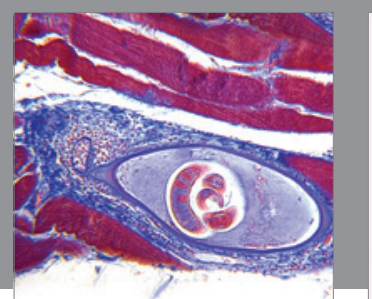

Gastroenterology Research and Practice

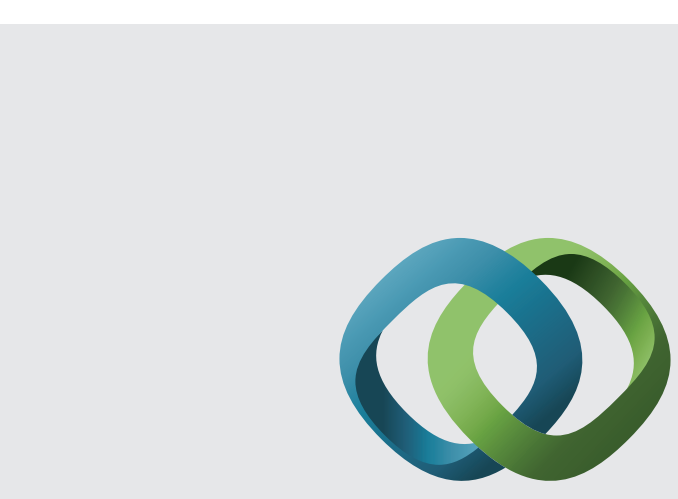

\section{Hindawi}

Submit your manuscripts at

http://www.hindawi.com
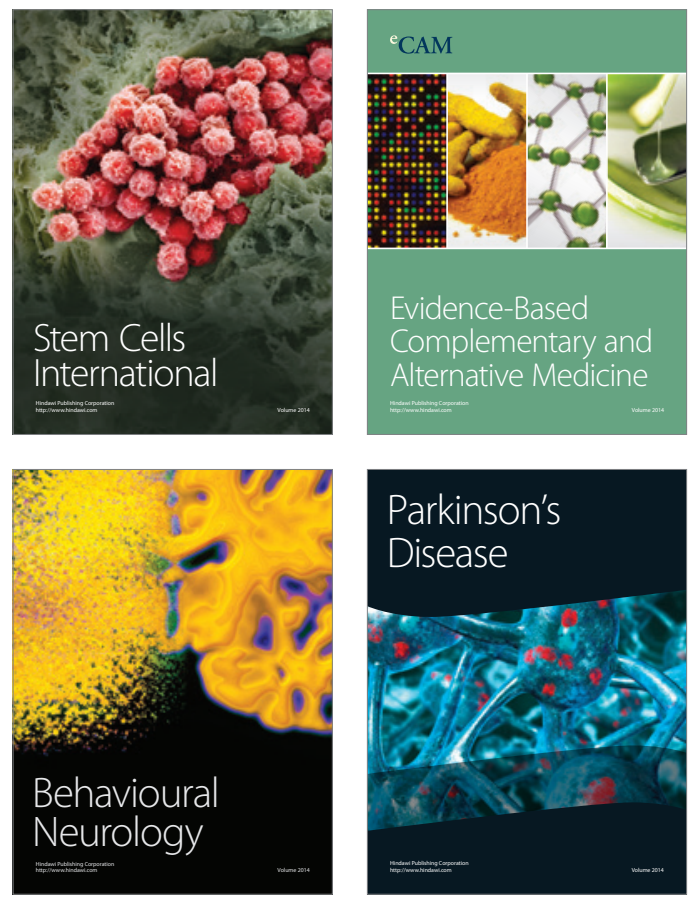
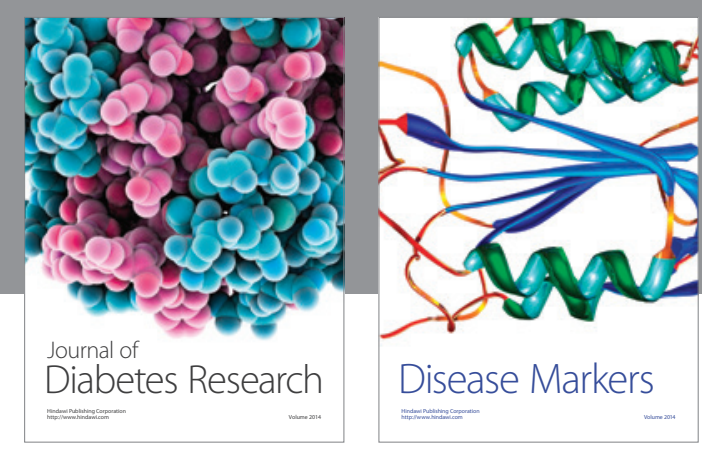

Disease Markers
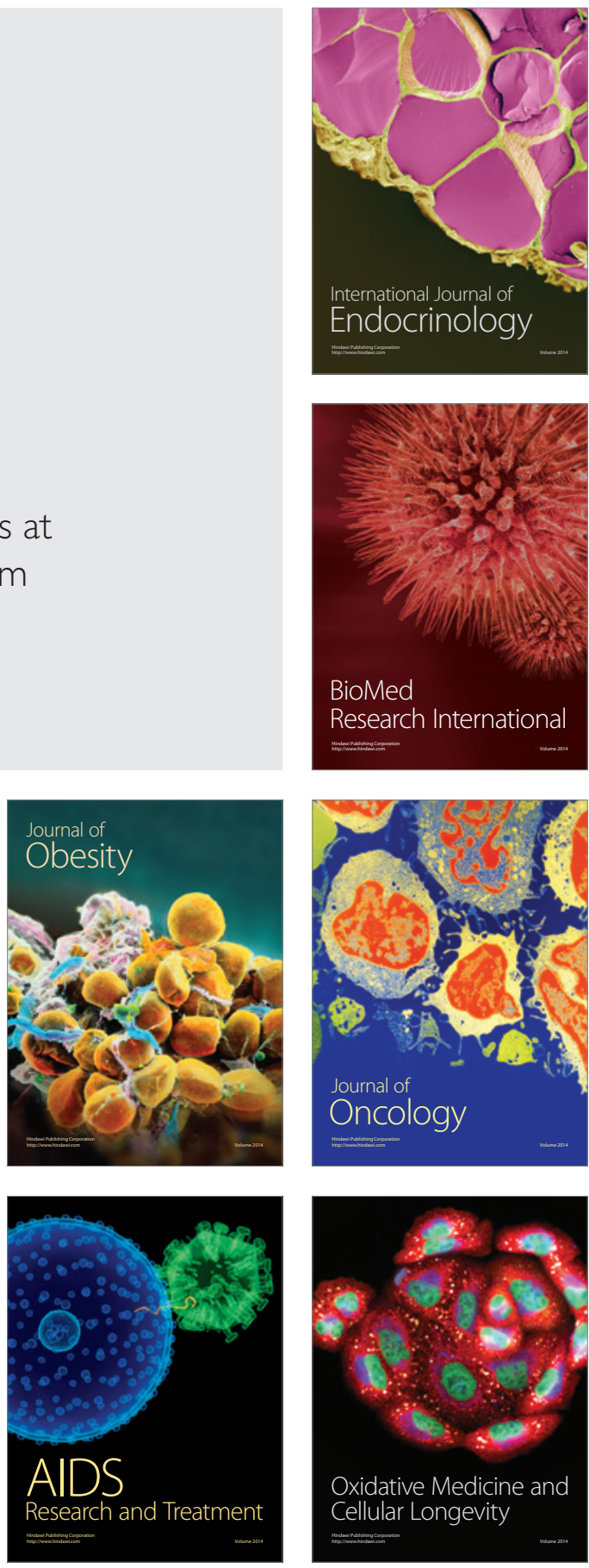